Article

\title{
Asymmetric Friedel-Crafts Alkylation of Indoles Catalyzed by Chiral Aziridine-Phosphines
}

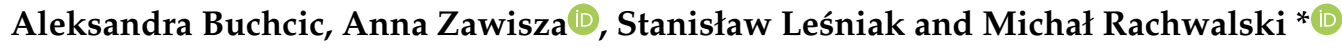 \\ Department of Organic and Applied Chemistry, University of Łódź, Tamka 12, 91-403 Łódź, Poland; \\ aleksandra.buchcic.ul@gmail.com (A.B.); anna.zawisza@chemia.uni.lodz.pl (A.Z.); \\ stanislaw.lesniak@chemia.uni.lodz.pl (S.L.) \\ * Correspondence: michal.rachwalski@chemia.uni.lodz.pl; Tel.: +48-42-635-5767
}

Received: 5 August 2020; Accepted: 24 August 2020; Published: 26 August 2020

\begin{abstract}
Over the course of the present studies, a series of optically pure phosphines functionalized by chiral aziridines was synthesized in reasonable/good chemical yields. Their catalytic activity was checked in the enantioselective Friedel-Crafts alkylation of indoles by $\beta$-nitrostyrene in the presence of a copper(I) trifluoromethanesulfonate benzene complex. The corresponding Friedel-Crafts products were achieved efficiently in terms of chemical yield and enantioselectivity (up to $85 \%$ in some cases).
\end{abstract}

Keywords: asymmetric transformations; chiral aziridinyl-phosphines; enantioselective Friedel-Crafts alkylation; stereoselectivity

\section{Introduction}

The synthesis of various organic molecules in an asymmetric manner remains one of the most important trends in modern synthetic organic chemistry. The desired chiral products of asymmetric transformations may be obtained using such approaches as organocatalysis [1,2], the use of chiral ligands or chiral auxiliary [3]. Additionally, more sophisticated methods like stereodivergent [4-6] or stereoconvergent synthesis [7-9] have been applied successfully in order to obtain various chiral systems.

The catalytic asymmetric Friedel-Crafts alkylation reaction [10] constitutes one of the most important methods of the enantioselective formation of carbon-carbon bonds. Many examples of catalysts for this transformation have been described in the chemical literature. These include bis(oxazoline)-Cu(II) [11] and bis(oxazoline)thiophenes-Cu(II) complexes [12], bifunctional thiourea-tertiary amine organocatalysts [13], cinchona alkaloids [14], a bisphenol A-derived Schiff base-Cu(II) complex [15], brucine-derived ligands [16] and metal-templated hydrogen bonding catalysts [17].

The enantioselective Friedel-Crafts reaction enables access to many valuable indole derivatives showing biological activity [18], like 2- and 3-substituted indoles [19], indoloquinolines [20] and spirooxindoles [21], and to the broad spectrum of other relevant molecules like dihydroisoquinolin3-ones [22], (+)-aflatoxin $B_{2}$ [23], Dalesconol A and B [24], steroids [25], dihydrocoumarins [26] and trinuclear ferrocenes [27].

Chiral phosphoroorganic compounds containing a three-membered ring of aziridine that act as ligands/organocatalysts in asymmetric synthesis are only rarely mentioned in the literature [28-32]. It should be pointed out that the successful application of phosphinoyl-aziridines as organocatalysts in the asymmetric Michael [33] and Mannich reaction [34] was reported in our department. Based on our experience in the field of asymmetric catalysis [35-37] and continuing our studies on phosphorus-containing chiral aziridines [33,34], we have attempted the synthesis of chiral aziridine-phosphines. Their catalytic ability was then checked in the asymmetric Friedel-Crafts alkylation of indoles using $\beta$-nitrostyrene in the presence of a copper(I) triflate benzene complex. 


\section{Results and Discussion}

\subsection{Synthesis of the Aziridine-Phosphines 1-8}

The chiral optically pure aziridine-phosphines 1-8 (Figure 1) were synthesized from the corresponding phosphine oxides.<smiles>CC(C)[C@H]1CN1Cc1ccccc1P</smiles>

$(R)-1$<smiles>CC(C)[C@H]1CN1c1ccccc1Pc1ccccc1</smiles>

$(R)-5$<smiles>CC(C)[C@H]1CN1Cc1ccccc1P</smiles>

$(S)-2$<smiles>CC(C)C1CN1c1ccccc1P</smiles>

$(S)-6$<smiles>CC(C)CC1CN1Cc1ccccc1P</smiles><smiles>Pc1ccccc1CN1CC1c1ccccc1</smiles>

$(S)-3$<smiles>Pc1cccc(N2CC2c2ccccc2)c1P</smiles>

$(S)-8$

Figure 1. Chiral aziridine-phosphines 1-8.

Systems 1-8 were obtained from the corresponding phosphine oxides using triethoxysilane and titanium(IV) isopropoxide in boiling tetrahydrofurane (Scheme 1) [38].

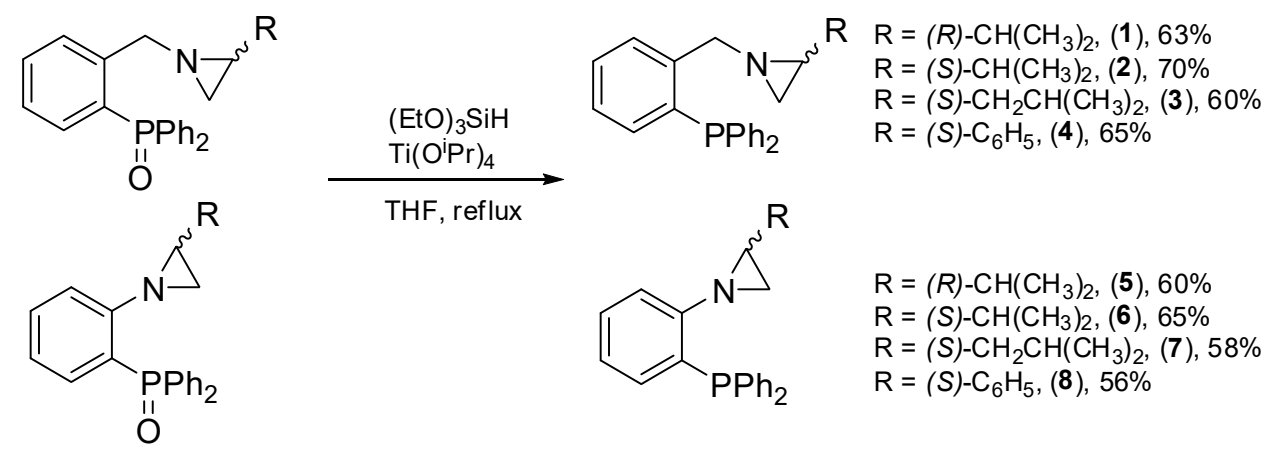

Scheme 1. Reduction of phosphine oxides to aziridine-phosphines 1-8.

The corresponding phosphine oxides were synthesized according to protocols reported by us previously [33,34]. It should be also mentioned that attempts at reduction of phosphine oxides using other reducing agents, e.g., Hantzsch ester [39], DIBAL-H [40], pinacol borane (HBpin) [41] or $\mathrm{BH}_{3}$ [42], turned out to be ineffective in our hands.

\subsection{Asymmetric Friedel-Crafts Alkylation of Indole Catalyzed by Aziridine Phosphines 1-8}

Having enantiomerically pure aziridine-phosphines $\mathbf{1 - 8}$, we decided to test their catalytic ability in the asymmetric Friedel-Crafts alkylation of indole with $\beta$-nitrostyrene in the presence of a $(\mathrm{CuOTf})_{2} \cdot \mathrm{C}_{6} \mathrm{H}_{6}$ complex and triethylamine in chloroform at $-15^{\circ} \mathrm{C}$ (Scheme 2) [16]. 


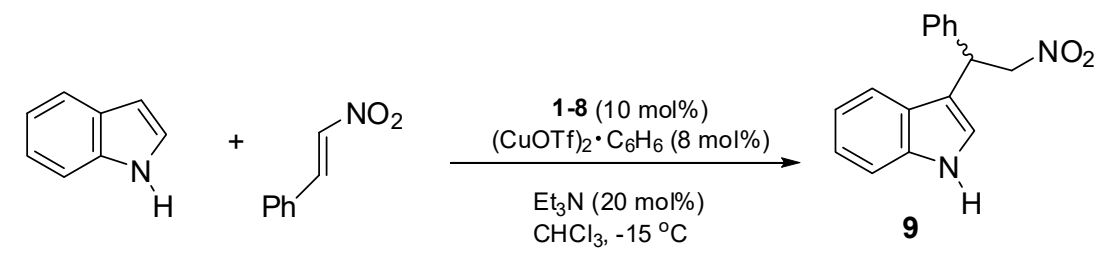

Scheme 2. Asymmetric Friedel-Crafts reaction promoted by aziridine-phosphines 1-8.

Chemical yields, enantiomeric excess (ee) values and absolute configurations of product 9 from the model asymmetric Friedel-Crafts alkylation reactions are summarized in Table 1.

Table 1. Asymmetric Friedel-Crafts alkylation of indole catalyzed by aziridine-phosphines 1-8.

\begin{tabular}{ccccc}
\hline Entry & Catalyst & Yield $[\%]$ & $e \boldsymbol{e}\left[\mathbf{\%}^{\mathbf{a}}\right.$ & Abs. Conf. ${ }^{\mathbf{b}}$ \\
\hline 1 & $\mathbf{1}$ & 40 & 30 & $(R)$ \\
2 & $\mathbf{2}$ & 39 & 30 & $(S)$ \\
3 & $\mathbf{3}$ & 36 & 27 & $(S)$ \\
4 & $\mathbf{4}$ & 33 & 24 & $(S)$ \\
5 & $\mathbf{5}$ & 69 & 84 & $(R)$ \\
6 & $\mathbf{6}$ & 75 & 80 & $(S)$ \\
7 & $\mathbf{7}$ & 68 & 68 & $(S)$ \\
8 & $\mathbf{8}$ & 65 & 68 & $(S)$
\end{tabular}

a Determined by chiral HPLC using a Chiralcel OD-H column (for the major product). ${ }^{\mathrm{b}}$ According to literature data [16]. Conditions: $10 \mathrm{~mol} \%$ of the catalyst, indole $(0.5 \mathrm{mmol}), \beta$-nitrostyrene $(0.5 \mathrm{mmol}),(\mathrm{CuOTf})_{2} \cdot \mathrm{C}_{6} \mathrm{H}_{6}$ $(8 \mathrm{~mol} \%), \mathrm{CHCl}_{3}(3 \mathrm{~mL}),-15^{\circ} \mathrm{C}, 48 \mathrm{~h}$.

Inspection of Table 1 reveals that aziridine-phosphines 1-4 bearing a methylene subunit between the aromatic ring and a nitrogen atom of aziridine exhibited relatively low catalytic activity leading to the Friedel-Crafts product 9 in yields from 33 to $40 \%$ and with enantioselectivity ranging from 26 to $30 \%$ of $e e$ (Table 1, entries 1-4). Moreover, as previously observed in the case of the asymmetric Michael addition [33] and the enantioselective Mannich reaction [34], the application of enantiomeric forms of the catalyst allowed for product 9 to be obtained in both enantiomeric forms (Table 1, entries 1 and 2). In turn, aziridine-phosphines 5-8 catalyzed the title reaction more effectively (Table 1 , entries 5-8), leading to 3-substituted indole 9 in $65-75 \%$ yields and with $68-84 \% e e$ 's. As previously, both enantiomeric products 9 can be achieved by the use of opposite enantiomers of the catalyst (Table 1, entries 5 and 6).

\subsection{Asymmetric Friedel-Crafts Reaction Catalyzed by Aziridine-Phosphine 6}

After screening the catalysts, we decided to check the influence of various additives on the course of the title asymmetric reaction (Scheme 3).

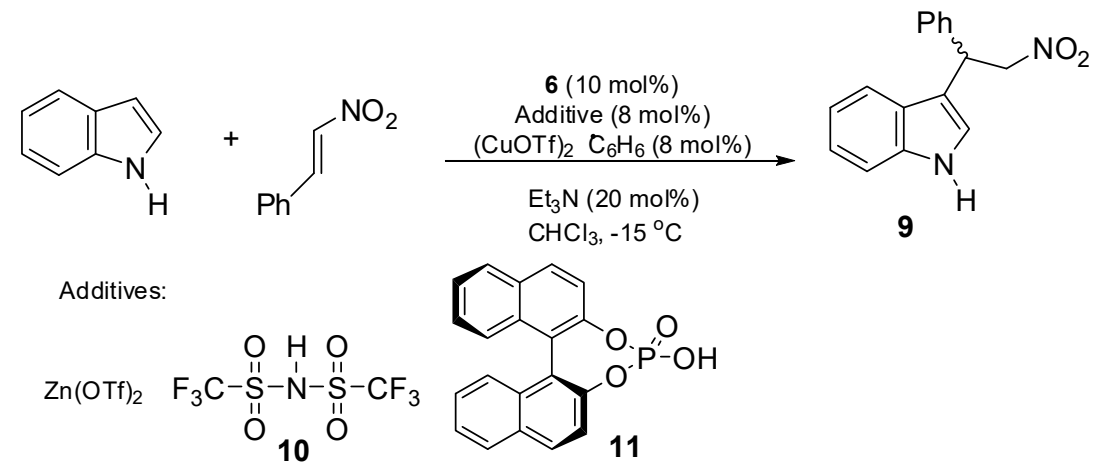

Scheme 3. Asymmetric Friedel-Crafts reaction catalyzed by aziridine-phosphine 6. 
Thus, zinc trifluoromethanesulfonate (instead of $(\mathrm{CuOTf})_{2} \cdot \mathrm{C}_{6} \mathrm{H}_{6}$ complex), triflimide 10 [43] and (S)-1,1'-binaphthyl-2,2'-diyl hydrogenphosphate 11 [44] were used in the asymmetric Friedel-Crafts alkylation of indole. The results are summarized in Table 2.

Table 2. Asymmetric Friedel-Crafts reaction catalyzed by aziridine-phosphine 6.

\begin{tabular}{ccccc}
\hline Entry & Additive & Yield [\%] & ee [\%] & Abs. Conf. $^{\mathbf{b}}$ \\
\hline 1 & $\mathrm{Zn}(\mathrm{OTf})_{2}{ }^{\mathrm{c}}$ & 60 & 56 & $(S)$ \\
2 & $\mathbf{1 0}$ & 62 & 84 & $(S)$ \\
3 & $\mathbf{1 1}$ & 63 & 84 & $(S)$ \\
\hline
\end{tabular}

a Determined by chiral HPLC using a Chiralcel OD-H column (for the major product). ${ }^{\mathrm{b}}$ According to literature data [16]. Conditions: $10 \mathrm{~mol} \%$ of the catalyst 6 , indole $(0.5 \mathrm{mmol}), \beta$-nitrostyrene $(0.5 \mathrm{mmol})$, additive $(8 \mathrm{~mol} \%)$, $(\mathrm{CuOTf})_{2} \cdot \mathrm{C}_{6} \mathrm{H}_{6}(8 \mathrm{~mol} \%), \mathrm{CHCl}_{3}(3 \mathrm{~mL}),-15^{\circ} \mathrm{C}, 48 \mathrm{~h} .{ }^{\mathrm{c}}$ Instead of copper salt.

As can be seen in Table 2, the use of zinc triflate resulted in a lowering of both the yield and enantiomeric excess of product 9 (Table 2, entry 1). The Friedel-Crafts alkylations performed in the presence of triflimide $\mathbf{1 0}$ and chiral acid $\mathbf{1 1}$ ran with slightly higher enantioselectivity; however, their chemical yields were lower in comparison with the process promoted by the $(\mathrm{CuOTf})_{2} \cdot \mathrm{C}_{6} \mathrm{H}_{6}$ complex (Table 2, entries 2 and 3).

Finally, we decided to expand the range of substrates. For this purpose, the asymmetric Friedel-Crafts alkylations were carried out using variously substituted indoles and $\beta$-nitrostyrenes in the presence of aziridine-phosphine 6 and the (CuOTf) $2 \cdot \mathrm{C}_{6} \mathrm{H}_{6}$ complex (Scheme 4). The results are collected in Table 3.<smiles>[R]C=C[N+](=O)[O-]</smiles>

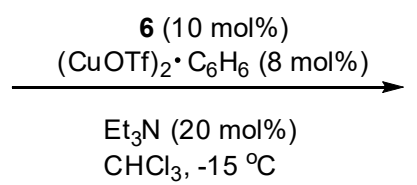<smiles>[R]Cc1c[nH]c2ccc([R7])cc12</smiles>

Scheme 4. Asymmetric Friedel-Crafts reaction of variously substituted substrates.

Table 3. Asymmetric Friedel-Crafts alkylation catalyzed by aziridine-phosphines 6.

\begin{tabular}{ccccccc}
\hline Entry & $\mathbf{R}^{\mathbf{1}}$ & $\mathbf{R}^{\mathbf{2}}$ & Product & Yield [\%] & ee [\%] & abs. Conf. \\
\hline 1 & $\mathrm{H}$ & $4-\mathrm{MeC}_{6} \mathrm{H}_{4}$ & $\mathbf{1 2}$ & 77 & 80 & $(S)$ \\
2 & $\mathrm{H}$ & $4-\mathrm{ClC}_{6} \mathrm{H}_{4}$ & $\mathbf{1 3}$ & 75 & 80 & $(S)$ \\
3 & $\mathrm{H}$ & $4-\mathrm{OMeC}_{6} \mathrm{H}_{4}$ & $\mathbf{1 4}$ & 80 & 84 & $(S)$ \\
4 & $\mathrm{H}$ & $3-\mathrm{ClC}_{6} \mathrm{H}_{4}$ & $\mathbf{1 5}$ & 72 & 80 & $(S)$ \\
5 & $\mathrm{OMe}$ & $\mathrm{Ph}$ & $\mathbf{1 6}$ & 85 & 88 & $(S)$ \\
6 & $\mathrm{Br}$ & $\mathrm{Ph}$ & $\mathbf{1 7}$ & 88 & 92 & $(S)$ \\
\hline
\end{tabular}

a Determined by chiral HPLC using a Chiralcel OD-H column. ${ }^{\mathrm{b}}$ According to literature data [16]. Conditions: $10 \mathrm{~mol} \%$ of the catalyst, indole $(0.5 \mathrm{mmol}), \beta$-nitrostyrene $(0.5 \mathrm{mmol}),(\mathrm{CuOTf})_{2} \cdot \mathrm{C}_{6} \mathrm{H}_{6}(8 \mathrm{~mol} \%), \mathrm{CHCl}_{3}(3 \mathrm{~mL})$, $-15^{\circ} \mathrm{C}, 48 \mathrm{~h}$.

The results in Table 3 clearly indicate that chiral aziridine-phosphine 6 worked well as a catalyst in the asymmetric Friedel-Crafts alkylation of indoles with $\beta$-nitrostyrenes in the presence of the $(\mathrm{CuOTf})_{2} \cdot \mathrm{C}_{6} \mathrm{H}_{6}$ complex and triethylamine, affording the corresponding chiral products $\mathbf{1 2 - 1 7}$ in yields and with enantioselectivity around $80 \%$. The best results were obtained in the reaction of 5 -bromoindole with $\beta$-nitrostyrene ( $88 \%$ yield and $92 \%$ of $e e$, respectively).

In our opinion, the possible transition state comprises the formation of an orthogonal system of catalyst-substrates analogous to the model proposed previously [16]. In this proposed mechanistic model, a minimalization of steric interactions takes place: the steric effect between the methylene $\mathrm{CH}_{2}$ 
group (C-2) of the aziridine ring and the phenyl ring of indole is almost insignificant in comparison with the steric hindrance induced in this place by the presence of a fragment of the ring containing an isopropyl group in the favorable trans-configuration relative to the substituent on the nitrogen atom (R-enantiomer) (Figure 2). Moreover, the Si-attack of indole on the double bond of styrene is more favorable, which is due to the quasi-trans orientation of the phenyl substituent of styrene to indole with respect to the bond being formed (Figure 2).

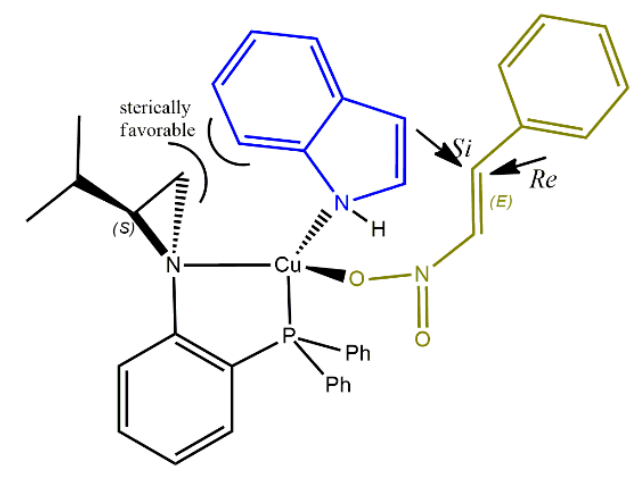

Figure 2. Tentative transition state model.

\section{Materials and Methods}

\subsection{Materials}

The drying of tetrahydrofurane was accomplished using the sodium benzophenone ketyl radical. Chloroform was distilled over phosphorus pentoxide. ${ }^{1} \mathrm{H},{ }^{13} \mathrm{C}$ and ${ }^{31} \mathrm{P}$ NMR spectra were recorded on a Bruker instrument at 600,150 and $243 \mathrm{MHz}$, respectively, with $\mathrm{CDCl}_{3}$ as solvent and TMS as the internal standard. Data are reported as $\mathrm{s}=$ singlet, $\mathrm{d}=$ doublet, $\mathrm{t}=$ triplet, $\mathrm{q}=$ quartet, $\mathrm{m}=$ multiplet, br. $\mathrm{s}=$ broad singlet. Column chromatography was conducted using Merck 60 silica gel. TLC was performed on Merck $60 \mathrm{~F}_{254}$ silica gel plates. The plates were visualized using UV light $(254 \mathrm{~nm})$ or iodine vapors. The enantiomeric excess (ee) values were determined by HPLC with chiral support (Chiralcel OD-H column). The corresponding chiral phosphinoyl-aziridines were synthesized according to general protocols reported by our group [33,34].

\subsection{Methods}

3.2.1. Reduction of Phosphinoyl-Aziridines to Aziridine-Phosphines 1-8-General Procedure (Coumbe, et al., 1994)

To a stirred mixture of phosphine oxide $(1 \mathrm{mmol})$ and triethoxysilane $(3 \mathrm{mmol})$ in dry THF $(5 \mathrm{~mL})$, titanium isopropoxide $(0.1 \mathrm{mmol})$ was added. The reaction mixture was refluxed until the completion of the reaction (TLC monitored) and cooled to room temperature. A solution of $\mathrm{NaOH}(1 \mathrm{M}, 10 \mathrm{~mL})$ was added. The resulting mixture was stirred for $2 \mathrm{~h}$ at room temperature and then extracted with ethyl acetate $(4 \times 15 \mathrm{~mL})$. The organic layer was dried with $\mathrm{MgSO}_{4}$ and evaporated in vacuo. The crude product was purified via column chromatography on silica gel (hexane:ethyl acetate 7:1).

(2R)-1-[2-(Diphenylphosphino)benzyl]-2-isopropylaziridine 1

Colorless oil, $63 \%$ yield; $[\alpha]_{\mathrm{D}}{ }^{20}=-4.2\left(c 0.5, \mathrm{CHCl}_{3}\right)$;

${ }^{1} \mathrm{H} \mathrm{NMR}\left(\mathrm{CDCl}_{3}, 600 \mathrm{MHz}\right): \delta=0.83(\mathrm{~d}, J=6.4 \mathrm{~Hz}, 3 \mathrm{H}), 0.86(\mathrm{~d}, J=6.4 \mathrm{~Hz}, 3 \mathrm{H}), 1.18-1.30(\mathrm{~m}, 3 \mathrm{H})$, $1.59(\mathrm{~d}, J=3.4 \mathrm{~Hz}, 1 \mathrm{H}), 3.54(\mathrm{~d}, J=14.5 \mathrm{~Hz}, 1 \mathrm{H}), 3.71(\mathrm{~d}, J=14.5 \mathrm{~Hz}, 1 \mathrm{H}), 6.83(\mathrm{dd}, J=7.5,4.6 \mathrm{~Hz}, 1 \mathrm{H})$, $7.14(\mathrm{t}, J=7.5 \mathrm{~Hz}, 1 \mathrm{H}), 7.20-7.27(\mathrm{~m}, 4 \mathrm{H}), 7.27-7.34(\mathrm{~m}, 6 \mathrm{H}), 7.37(\mathrm{t}, J=7.6 \mathrm{~Hz}, 1 \mathrm{H}), 7.77(\mathrm{dd}, J=7.5$, $4.3 \mathrm{~Hz}, 1 \mathrm{H})$;

${ }^{13} \mathrm{C} \mathrm{NMR}\left(150 \mathrm{MHz}, \mathrm{CDCl}_{3}\right): \delta=19.6\left(\mathrm{CH}_{3}\right), 20.7\left(\mathrm{CH}_{3}\right), 31.5(\mathrm{CHN}), 32.2(\mathrm{CHN}), 46.7\left(\mathrm{CH}_{\left(\mathrm{CH}_{3}\right)_{2}}\right.$, $62.6\left(\mathrm{~d}, J=22.9 \mathrm{~Hz}, \mathrm{CH}_{2} \mathrm{C}_{6} \mathrm{H}_{4}\right), 127.1\left(\mathrm{C}_{\mathrm{Ar}}\right), 128.5\left(\mathrm{~d}, J=5.5 \mathrm{~Hz}, \mathrm{C}_{\mathrm{Ar}}\right), 128.5\left(\mathrm{~d}, J=6.8 \mathrm{~Hz}, \mathrm{C}_{\mathrm{Ar}}\right), 128.8$ 
$\left(\mathrm{d}, J=4.4 \mathrm{~Hz}, \mathrm{C}_{\mathrm{Ar}}\right), 129.0,133.2\left(\mathrm{C}_{\mathrm{Ar}}\right), 134.0\left(\mathrm{~d}, J=13.6 \mathrm{~Hz}, \mathrm{C}_{\mathrm{Ar}}\right), 134.1\left(\mathrm{~d}, J=13.6 \mathrm{~Hz}, \mathrm{C}_{\mathrm{Ar}}\right), 134.9(\mathrm{~d}$,

$\left.J=13.1 \mathrm{~Hz}, \mathrm{C}_{\mathrm{Ar}}\right), 136.8\left(\mathrm{~d}, J=9.9 \mathrm{~Hz}, \mathrm{C}_{\mathrm{Ar}}\right), 144.3\left(\mathrm{~d}, J=23.1 \mathrm{~Hz}, \mathrm{C}_{\mathrm{Ar}}\right)$;

${ }^{31} \mathrm{P} \mathrm{NMR}\left(243 \mathrm{MHz}, \mathrm{CDCl}_{3}\right): \delta=-15.63$;

Anal. Calcd. for $\mathrm{C}_{24} \mathrm{H}_{26} \mathrm{NP}$ C, 80.20; H, 7.29; N, 3.90; Found: C, 80.13; H, 7.33; N, 3.75.

(2S)-1-[2-(Diphenylphosphino)benzyl]-2-isopropylaziridine 2

Colorless oil, $70 \%$ yield; $[\alpha]_{\mathrm{D}}{ }^{20}=+5.2\left(c 0.5, \mathrm{CHCl}_{3}\right)$;

${ }^{1} \mathrm{H} \mathrm{NMR}\left(\mathrm{CDCl}_{3}, 600 \mathrm{MHz}\right): \delta=0.83(\mathrm{~d}, J=6.5 \mathrm{~Hz}, 3 \mathrm{H}), 0.86(\mathrm{~d}, J=6.5 \mathrm{~Hz}, 3 \mathrm{H}), 1.18-1.30(\mathrm{~m}, 3 \mathrm{H})$, $1.59(\mathrm{~d}, J=3.3 \mathrm{~Hz}, 1 \mathrm{H}), 3.56(\mathrm{~d}, J=14.5 \mathrm{~Hz}, 1 \mathrm{H}), 3.72(\mathrm{~d}, J=14.5 \mathrm{~Hz}, 1 \mathrm{H}), 6.83(\mathrm{dd}, \mathrm{J}=7.0,4.7 \mathrm{~Hz}, 1 \mathrm{H})$, $7.14(\mathrm{t}, J=7.6 \mathrm{~Hz}, 1 \mathrm{H}), 7.19-7.27(\mathrm{~m}, 4 \mathrm{H}), 7.27-7.34(\mathrm{~m}, 6 \mathrm{H}), 7.36(\mathrm{t}, J=7.4 \mathrm{~Hz}, 1 \mathrm{H}), 7.77(\mathrm{dd}, J=7.4$, $4.4 \mathrm{~Hz}, 1 \mathrm{H})$;

${ }^{13} \mathrm{C} \mathrm{NMR}\left(150 \mathrm{MHz}, \mathrm{CDCl}_{3}\right): \delta=19.6\left(\mathrm{CH}_{3}\right), 20.7\left(\mathrm{CH}_{3}\right), 31.4(\mathrm{CHN}), 33.2(\mathrm{CHN}), 46.7\left(\mathrm{CH}\left(\mathrm{CH}_{3}\right)_{2}\right.$, $62.6\left(\mathrm{~d}, J=22.9 \mathrm{~Hz}, \mathrm{CH}_{2} \mathrm{C}_{6} \mathrm{H}_{4}\right), 127.1\left(\mathrm{C}_{\mathrm{Ar}}\right), 128.5\left(\mathrm{~d}, J=5.4 \mathrm{~Hz}, \mathrm{C}_{\mathrm{Ar}}\right), 128.6\left(\mathrm{~d}, J=6.8 \mathrm{~Hz}, \mathrm{C}_{\mathrm{Ar}}\right), 128.8$ $\left(\mathrm{d}, J=4.4 \mathrm{~Hz}, \mathrm{C}_{\mathrm{Ar}}\right), 129.0,132.2\left(\mathrm{C}_{\mathrm{Ar}}\right), 134.0\left(\mathrm{~d}, J=13.7 \mathrm{~Hz}, \mathrm{C}_{\mathrm{Ar}}\right), 134.1\left(\mathrm{~d}, J=13.6 \mathrm{~Hz}, \mathrm{C}_{\mathrm{Ar}}\right), 134.9(\mathrm{~d}$, $\left.J=13.2 \mathrm{~Hz}, \mathrm{C}_{\mathrm{Ar}}\right), 136.8\left(\mathrm{~d}, J=9.9 \mathrm{~Hz}, \mathrm{C}_{\mathrm{Ar}}\right), 144.3\left(\mathrm{~d}, J=23.2 \mathrm{~Hz}, \mathrm{C}_{\mathrm{Ar}}\right)$;

${ }^{31} \mathrm{P}$ NMR $\left(243 \mathrm{MHz}, \mathrm{CDCl}_{3}\right): \delta=-15.65$;

Anal. Calcd. for $\mathrm{C}_{24} \mathrm{H}_{26} \mathrm{NP}$ C, 80.20; H, 7.29; N, 3.90; Found: C, 80.27; H, 7.28; N, 3.90.

(2S)-1-[2-(Diphenylphosphino)benzyl]-2-isobutylaziridine 3

Colorless oil, $60 \%$ yield; $[\alpha]_{\mathrm{D}}{ }^{20}=+13.4\left(c 0.5, \mathrm{CHCl}_{3}\right)$;

${ }^{1} \mathrm{H} \mathrm{NMR}\left(\mathrm{CDCl}_{3}, 600 \mathrm{MHz}\right): \delta=0.85(\mathrm{~d}, J=6.7 \mathrm{~Hz}, 3 \mathrm{H}), 0.87(\mathrm{~d}, J=6.7 \mathrm{~Hz}, 3 \mathrm{H}), 1.05-1.15(\mathrm{~m}, 1 \mathrm{H})$, $1.32(\mathrm{~d}, J=6.3 \mathrm{~Hz}, 1 \mathrm{H}), 1.40-1.48(\mathrm{~m}, 2 \mathrm{H}), 1.54(\mathrm{~d}, J=3.1 \mathrm{~Hz}, 1 \mathrm{H}), 1.60-1.68(\mathrm{~m}, 1 \mathrm{H}), 3.57(\mathrm{~d}, J=14.7 \mathrm{~Hz}$, $1 \mathrm{H}), 3.69(\mathrm{~d}, J=14.7 \mathrm{~Hz}, 1 \mathrm{H}), 6.82(\mathrm{dd}, J=7.4,4.7 \mathrm{~Hz}, 1 \mathrm{H}), 7.14(\mathrm{t}, J=7.3 \mathrm{~Hz}, 1 \mathrm{H}), 7.20-7.26(\mathrm{~m}, 4 \mathrm{H})$, $7.28-7.35(\mathrm{~m}, 6 \mathrm{H}), 7.37(\mathrm{t}, J=7.6 \mathrm{~Hz}, 1 \mathrm{H}), 7.76(\mathrm{dd}, J=7.6,4.4 \mathrm{~Hz}, 1 \mathrm{H})$;

${ }^{13} \mathrm{C} \mathrm{NMR}\left(150 \mathrm{MHz}, \mathrm{CDCl}_{3}\right): \delta=22.5\left(\mathrm{CH}_{3}\right), 23.1\left(\mathrm{CH}_{3}\right), 27.3\left(\mathrm{CH}\left(\mathrm{CH}_{3}\right)_{2}, 34.7(\mathrm{CHN}), 38.7(\mathrm{CHN})\right.$, $42.3\left(\mathrm{CH}_{2} \mathrm{CH}\left(\mathrm{CH}_{3}\right)_{2}, 62.3\left(\mathrm{~d}, J=23.1 \mathrm{~Hz}, \mathrm{CH}_{2} \mathrm{C}_{6} \mathrm{H}_{4}\right), 127.0\left(\mathrm{C}_{\mathrm{Ar}}\right), 128.1\left(\mathrm{~d}, J=5.4 \mathrm{~Hz}, \mathrm{C}_{\mathrm{Ar}}\right), 128.5,128.6\right.$, $128.8,129.1,133.1\left(\mathbf{C}_{\mathrm{Ar}}\right), 134.0\left(\mathrm{~d}, J=7.6 \mathrm{~Hz}, \mathrm{C}_{\mathrm{Ar}}\right), 134.1\left(\mathrm{~d}, J=7.6 \mathrm{~Hz}, \mathrm{C}_{\mathrm{Ar}}\right), 134.7\left(\mathrm{~d}, J=13.4 \mathrm{~Hz}, \mathrm{C}_{\mathrm{Ar}}\right)$, $136.6\left(\mathrm{~d}, J=9.9 \mathrm{~Hz}, \mathrm{C}_{\mathrm{Ar}}\right), 144.2\left(\mathrm{~d}, J=23.0 \mathrm{~Hz}, \mathrm{C}_{\mathrm{Ar}}\right)$;

${ }^{31} \mathrm{P} \mathrm{NMR}\left(243 \mathrm{MHz}, \mathrm{CDCl}_{3}\right): \delta=-15.60$;

Anal. Calcd. for $\mathrm{C}_{25} \mathrm{H}_{28} \mathrm{NP}$ C, 80.40; H, 7.56; N, 3.75; Found: C, 80.26; H, 7.49; N, 3.81 .

(S)-1-\{2-(Diphenylphosphino)benzyl\}-2-phenylaziridine 4

Colorless oil, $65 \%$ yield; $[\alpha]_{\mathrm{D}}{ }^{20}=+100.1\left(c 0.5, \mathrm{CHCl}_{3}\right)$;

${ }^{1} \mathrm{H} \mathrm{NMR}\left(\mathrm{CDCl}_{3}, 600 \mathrm{MHz}\right): \delta=1.77(\mathrm{~d}, J=6.5 \mathrm{~Hz}, 1 \mathrm{H}), 1.87(\mathrm{~d}, J=3.3 \mathrm{~Hz}, 1 \mathrm{H}), 2.40-2.43(\mathrm{~m}, 1 \mathrm{H})$, $3.80(\mathrm{~d}, J=14.8 \mathrm{~Hz}, 1 \mathrm{H}), 3.85(\mathrm{~d}, J=14.8 \mathrm{~Hz}, 1 \mathrm{H}), 6.83(\mathrm{dd}, J=7.4,4.6 \mathrm{~Hz}, 1 \mathrm{H}), 7.18(\mathrm{t}, J=7.5 \mathrm{~Hz}, 1 \mathrm{H})$, 7.15-7.21 (m, 2H), 7.21-7.27 (m, 6H), 7.28-7.35 (m, 8H), $7.55(\mathrm{dd}, J=7.5,4.4 \mathrm{~Hz}, 1 \mathrm{H})$;

${ }^{13} \mathrm{C}$ NMR $\left(150 \mathrm{MHz}, \mathrm{CDCl}_{3}\right): \delta=38.5(\mathrm{CHN}), 41.8(\mathrm{CHN}), 62.7\left(\mathrm{~d}, J=23.7 \mathrm{~Hz}, \mathrm{CH}_{2} \mathrm{C}_{6} \mathrm{H}_{4}\right), 126.3$, $126.9,127.1\left(\mathrm{C}_{\mathrm{Ar}}\right), 128.0\left(\mathrm{~d}, J=5.4 \mathrm{~Hz}, \mathrm{C}_{\mathrm{Ar}}\right), 128.4\left(\mathrm{C}_{\mathrm{Ar}}\right), 128.6\left(\mathrm{~d}, J=6.3 \mathrm{~Hz}, \mathrm{C}_{\mathrm{Ar}}\right), 128.7(\mathrm{~d}, J=6.2 \mathrm{~Hz}$, $\left.\mathrm{C}_{\mathrm{Ar}}\right), 128.6\left(\mathrm{~d}, J=3.3 \mathrm{~Hz}, \mathrm{C}_{\mathrm{Ar}}\right), 129.2,133.3\left(\mathrm{C}_{\mathrm{Ar}}\right), 134.0\left(\mathrm{~d}, J=2.0 \mathrm{~Hz}, \mathrm{C}_{\mathrm{Ar}}\right), 134.1\left(\mathrm{~d}, J=2.0 \mathrm{~Hz}, \mathrm{C}_{\mathrm{Ar}}\right)$, $134.8\left(\mathrm{~d}, J=14.1 \mathrm{~Hz}, \mathrm{C}_{\mathrm{Ar}}\right), 136.6\left(\mathrm{~d}, J=9.6 \mathrm{~Hz}, \mathrm{C}_{\mathrm{Ar}}\right), 140.5\left(\mathrm{C}_{\mathrm{Ar}}\right), 143.8\left(\mathrm{~d}, J=22.9 \mathrm{~Hz}, \mathrm{C}_{\mathrm{Ar}}\right)$;

${ }^{31} \mathrm{P} \mathrm{NMR}\left(\mathrm{CDCl}_{3}, 243 \mathrm{MHz}\right): \delta=-15.78$;

Anal. Calcd. for $\mathrm{C}_{27} \mathrm{H}_{24} \mathrm{NP}$ C, 82.42; H, 6.15; N, 3.56; Found: C, 82.53; H, 6.26; N, 3.52 .

(2R)-1-[2-(Diphenylphosphinophenyl]-2-isopropylaziridine 5

Colorless oil, $60 \%$ yield; $[\alpha]_{\mathrm{D}}^{20}=-60.2\left(\right.$ c $\left.0.5, \mathrm{CHCl}_{3}\right)$;

${ }^{1} \mathrm{H} \mathrm{NMR}\left(\mathrm{CDCl}_{3}, 600 \mathrm{MHz}\right): \delta=0.87(\mathrm{~d}, J=6.7 \mathrm{~Hz}, 3 \mathrm{H}), 1.05(\mathrm{~d}, J=6.7 \mathrm{~Hz}, 3 \mathrm{H}), 1.72-1.77(\mathrm{~m}, 1 \mathrm{H})$, $1.77-1.80(\mathrm{~m}, 1 \mathrm{H}), 2.08-2.11(\mathrm{~m}, 2 \mathrm{H}), 6.76-6.80(\mathrm{~m}, 1 \mathrm{H}), 6.88-6.91(\mathrm{~m}, 1 \mathrm{H}), 6.93-6.97(\mathrm{~m}, 1 \mathrm{H}), 7.24-7.27$ $(\mathrm{m}, 1 \mathrm{H}), 7.27-7.31(\mathrm{~m}, 2 \mathrm{H}), 7.31-7.34(\mathrm{~m}, 3 \mathrm{H}), 7.35-7.40(\mathrm{~m}, 5 \mathrm{H})$;

${ }^{13} \mathrm{C} \mathrm{NMR}\left(\mathrm{CDCl}_{3}, 150 \mathrm{MHz}\right): \delta=18.5\left(\mathrm{CH}_{3}\right), 20.7\left(\mathrm{CH}_{3}\right), 30.1(\mathrm{~d}, J=1.8 \mathrm{~Hz}, \mathrm{CH}), 34.6(\mathrm{~d}, J=6.8 \mathrm{~Hz}$, $\left.\mathrm{CH}_{2} \mathrm{~N}\right), 46.3(\mathrm{~d}, J=5.5 \mathrm{~Hz}, \mathrm{CHN}), 119.4\left(\mathrm{~d}, J=3.4 \mathrm{~Hz}, \mathrm{C}_{\mathrm{ar}}\right), 122.3\left(\mathrm{C}_{\mathrm{ar}}\right), 128.2,\left(\mathrm{C}_{\mathrm{ar}}\right), 128.4\left(\mathrm{C}_{\mathrm{ar}}\right), 128.5$ $\left(C_{a r}\right), 128.7\left(C_{a r}\right), 129.6\left(C_{a r}\right), 133.8\left(C_{a r}\right), 133.9\left(C_{a r}\right), 134.2\left(C_{a r}\right), 134.3\left(C_{a r}\right), 134.4\left(C_{a r}\right), 137.0\left(C_{a r}\right)$, $137.1\left(\mathbf{C}_{\mathrm{ar}}\right), 137.2\left(\mathrm{C}_{\mathrm{ar}}\right), 137.3\left(\mathrm{C}_{\mathrm{ar}}\right), 157.7\left(\mathrm{C}_{\mathrm{q} \text { ar }}\right), 157.8\left(\mathrm{C}_{\mathrm{q} \text { ar }}\right)$;

${ }^{31} \mathrm{P}$ NMR $\left(243 \mathrm{MHz}, \mathrm{CDCl}_{3}\right): \delta=-17.3$;

Anal. Calcd. for $\mathrm{C}_{23} \mathrm{H}_{24} \mathrm{NP}$ C, 80.00; H, 7.00; N, 4.10; Found: C, 80.05; H, 7.21; N, 4.11. 
(2S)-1-[2-(Diphenylphosphinophenyl]-2-isopropylaziridine 6

Colorless oil, $65 \%$ yield; $[\alpha]_{\mathrm{D}}{ }^{20}=+21.3\left(c 0.5, \mathrm{CHCl}_{3}\right)$;

${ }^{1} \mathrm{H} \mathrm{NMR}\left(\mathrm{CDCl}_{3}, 600 \mathrm{MHz}\right): \delta=0.87(\mathrm{~d}, J=6.7 \mathrm{~Hz}, 3 \mathrm{H}), 1.06(\mathrm{~d}, J=6.7 \mathrm{~Hz}, 3 \mathrm{H}), 1.71-1.75(\mathrm{~m}, 1 \mathrm{H})$, $1.77-1.80(\mathrm{~m}, 1 \mathrm{H}), 2.07-2.11(\mathrm{~m}, 2 \mathrm{H}), 6.76-6.80(\mathrm{~m}, 1 \mathrm{H}), 6.88-6.91(\mathrm{~m}, 1 \mathrm{H}), 6.93-6.97(\mathrm{~m}, 1 \mathrm{H}), 7.23-7.27$ $(\mathrm{m}, 1 \mathrm{H}), 7.28-7.31(\mathrm{~m}, 2 \mathrm{H}), 7.31-7.34(\mathrm{~m}, 3 \mathrm{H}), 7.36-7.41(\mathrm{~m}, 5 \mathrm{H})$;

${ }^{13} \mathrm{C} \mathrm{NMR}\left(\mathrm{CDCl}_{3}, 150 \mathrm{MHz}\right): \delta=18.5\left(\mathrm{CH}_{3}\right), 20.8\left(\mathrm{CH}_{3}\right), 30.1(\mathrm{CH}), 34.8\left(\mathrm{~d}, J=6.8 \mathrm{~Hz}, \mathrm{CH}_{2} \mathrm{~N}\right)$, $46.3(\mathrm{~d}, J=5.5 \mathrm{~Hz}, \mathrm{CHN}), 119.4\left(\mathrm{~d}, J=3.2 \mathrm{~Hz}, \mathrm{C}_{\mathbf{a r}}\right), 122.3\left(\mathrm{C}_{\mathrm{ar}}\right), 128.3\left(2 \mathrm{C}_{\mathbf{a r}}\right), 128.4\left(\mathrm{C}_{\mathbf{a r}}\right), 128.7\left(\mathrm{C}_{\mathrm{ar}}\right)$, $129.6\left(\mathbf{C}_{\mathrm{ar}}\right), 133.8\left(\mathrm{C}_{\mathrm{ar}}\right), 133.9\left(\mathbf{C}_{\mathrm{ar}}\right), 134.2\left(\mathbf{C}_{\mathrm{ar}}\right), 134.4\left(\mathbf{C}_{\mathrm{ar}}\right), 137.0\left(\mathbf{C}_{\mathrm{ar}}\right), 137.1\left(\mathbf{C}_{\mathrm{ar}}\right), 137.2\left(\mathbf{C}_{\mathrm{ar}}\right), 137.3$ $\left(\mathbf{C}_{\mathrm{ar}}\right), 157.7\left(\mathrm{C}_{\mathrm{q} \text { ar }}\right), 157.8\left(\mathrm{C}_{\mathrm{q} \text { ar }}\right)$;

${ }^{31} \mathrm{P} \mathrm{NMR}\left(\mathrm{CDCl}_{3}, 243 \mathrm{MHz}\right): \delta=-17.3$;

Anal. Calcd. for $\mathrm{C}_{23} \mathrm{H}_{24} \mathrm{NP}$ C, 80.00; H, 7.00; N, 4.10; Found: C, 79.95; H, 6.98; N, 4.12.

(2S)-1-[2-(Diphenylphosphinophenyl]-2-isobutylaziridine 7

Colorless oil, $58 \%$ yield; $[\alpha]_{\mathrm{D}}{ }^{20}=+32.6\left(c 0.5, \mathrm{CHCl}_{3}\right)$;

${ }^{1} \mathrm{H} \mathrm{NMR}\left(\mathrm{CDCl}_{3}, 600 \mathrm{MHz}\right): \delta=0.88(\mathrm{~d}, J=6.7 \mathrm{~Hz}, 3 \mathrm{H}), 0.92(\mathrm{~d}, J=6,7 \mathrm{~Hz}, 3 \mathrm{H}), 1.07-1.12(\mathrm{~m}, 1 \mathrm{H})$, 1.62-1.7 (m, 1H), 1.88-1.94 (m, 1H), $2.04(\mathrm{~m}, 2 \mathrm{H}), 2.09-2.14(\mathrm{~m}, 1 \mathrm{H}), 6.78-6.81(\mathrm{~m}, 1 \mathrm{H}), 6.89-6.95(\mathrm{~m}$, $2 \mathrm{H}), 7.25-7.28(\mathrm{~m}, 1 \mathrm{H}), 7.29-7.32(\mathrm{~m}, 2 \mathrm{H}), 7.32-7.38(\mathrm{~m}, 8 \mathrm{H})$;

${ }^{13} \mathrm{C} \mathrm{NMR}\left(\mathrm{CDCl}_{3}, 150 \mathrm{MHz}\right): \delta=22.6\left(\mathrm{CH}_{3}\right), 22.7\left(\mathrm{CH}_{3}\right), 26.9(\mathrm{CH}), 35.7\left(\mathrm{~d}, J=5.5 \mathrm{~Hz}, \mathrm{CH}_{2}\right), 39.7$ $\left(\mathrm{d}, J=4.7 \mathrm{~Hz}, \mathrm{CH}_{2} \mathrm{~N}\right), 41.4(\mathrm{~d}, J=3.3 \mathrm{~Hz}, \mathrm{CHN}), 119.6\left(\mathrm{C}_{\mathbf{a r}}\right), 122.4\left(\mathrm{C}_{\mathbf{a r}}\right), 128.3,\left(\mathrm{C}_{\mathrm{ar}}\right), 128.4\left(2 \mathrm{C}_{\mathrm{ar}}\right), 128.5$ $\left(C_{a r}\right), 128.6\left(C_{a r}\right), 129.7\left(C_{a r}\right), 129.8\left(C_{a r}\right), 133.8\left(C_{a r}\right), 134.0\left(C_{a r}\right), 134.1\left(C_{a r}\right), 134.4\left(C_{a r}\right), 137.1\left(C_{a r}\right)$, $137.3\left(\mathrm{C}_{\mathrm{ar}}\right), 157.4\left(\mathrm{C}_{\mathrm{q} \text { ar }}\right), 157.6\left(\mathrm{C}_{\mathrm{q} \text { ar }}\right)$;

${ }^{31} \mathrm{P} \mathrm{NMR}\left(\mathrm{CDCl}_{3}, 243 \mathrm{MHz}\right): \delta=-17.3$;

Anal. Calcd. for $\mathrm{C}_{24} \mathrm{H}_{26} \mathrm{NP}$ C, 80.20; H, 7.30; N, 3.90; Found: C, 80.37; H, 7.14; N, 3.74.

(2S)-1-[2-(Diphenylphosphinophenyl]-2-phenylaziridine 8

Colorless oil, $56 \%$ yield; $[\alpha]_{\mathrm{D}}{ }^{20}=+25.8\left(c 0.5, \mathrm{CHCl}_{3}\right)$;

${ }^{1} \mathrm{H}$ NMR $\left(\mathrm{CDCl}_{3}, 600 \mathrm{MHz}\right): \delta=2.32(\mathrm{~d}, J=3.2 \mathrm{~Hz}, 1 \mathrm{H}), 2.54(\mathrm{~d}, J=6.4 \mathrm{~Hz}, 1 \mathrm{H}), 3.19(\mathrm{dd}, J=3.2$, $6.3 \mathrm{~Hz}, 1 \mathrm{H}), 6.75-6.79(\mathrm{~m}, 1 \mathrm{H}), 6.93-6.97(\mathrm{~m}, 1 \mathrm{H}), 7.00-7.04(\mathrm{~m}, 1 \mathrm{H}), 7.08-7.12(\mathrm{~m}, 2 \mathrm{H}), 7.16-7.20(\mathrm{~m}$, 2H), 7.21-7.25 (m, 5H), 7.28-7.37 (m, 6H), $7.39(\mathrm{~s}, 1 \mathrm{H})$;

${ }^{13} \mathrm{C} \mathrm{NMR}\left(\mathrm{CDCl}_{3}, 150 \mathrm{MHz}\right): \delta=39.3\left(\mathrm{~d}, J=5.6 \mathrm{~Hz}, \mathrm{CH}_{2} \mathrm{~N}\right), 42.5(\mathrm{~d}, J=6.6 \mathrm{~Hz}, \mathrm{CHN}), 119.2$ $\left(C_{a r}\right), 119.3\left(C_{a r}\right), 122.7\left(C_{a r}\right), 126.1\left(C_{a r}\right), 126.9\left(C_{a r}\right), 128.0\left(C_{a r}\right), 128.2\left(C_{a r}\right), 128.3\left(C_{a r}\right), 128.4\left(C_{a r}\right)$, $128.5\left(\mathbf{C}_{\mathbf{a r}}\right), 129.5\left(\mathbf{C}_{\mathbf{a r}}\right), 133.8\left(\mathbf{C}_{\mathbf{a r}}\right), 134.0\left(\mathbf{C}_{\mathbf{a r}}\right), 134.2\left(\mathbf{C}_{\mathbf{a r}}\right), 134.3\left(\mathbf{C}_{\mathbf{a r}}\right), 136.3\left(\mathbf{C}_{\mathbf{a r}}\right), 136.4\left(\mathbf{C}_{\mathbf{a r}}\right), 136.6\left(\mathbf{C}_{\mathbf{a r}}\right)$, $139.1\left(\mathrm{C}_{\mathrm{ar}}\right), 156.0\left(\mathrm{C}_{\mathrm{q} \text { ar }}\right), 156.1\left(\mathrm{C}_{\mathrm{q} \text { ar }}\right)$;

${ }^{31} \mathrm{P} \mathrm{NMR}\left(\mathrm{CDCl}_{3}, 243 \mathrm{MHz}\right): \delta=-15.9$;

Anal. Calcd. for $\mathrm{C}_{26} \mathrm{H}_{22} \mathrm{NP} C, 82.30 ; \mathrm{H}, 5.80 ; \mathrm{N}$, 3.70; Found: $\mathrm{C}, 82.26 ; \mathrm{H}, 5.83 ; \mathrm{N}, 3.67$.

\subsubsection{Asymmetric Friedel-Crafts Alkylation of Indoles-General Procedure (Kim, et al., 2010)}

A solution of $(\mathrm{CuOTf})_{2} \cdot \mathrm{C}_{6} \mathrm{H}_{6}(8 \mathrm{~mol} \%, 0.04 \mathrm{mmol})$, chiral ligand $(10 \mathrm{~mol} \%, 0.05 \mathrm{mmol})$ and triethylamine $(0.1 \mathrm{mmol})$ in chloroform $(3 \mathrm{~mL})$ was stirred at $0{ }^{\circ} \mathrm{C}$ for $4 \mathrm{~h}$. After the generation of the catalyst, trans- $\beta$-nitrostyrene $(0.5 \mathrm{mmol})$ and indole $(0.5 \mathrm{mmol})$ were added and stirred at $-15^{\circ} \mathrm{C}$ for $48 \mathrm{~h}$. The reaction mixture was directly loaded on the column and chromatographed on silica gel (hexane:ethyl acetate 95:5 $\rightarrow$ 80:20) to afford the corresponding Friedel-Crafts products. Their NMR spectra are consistent with literature data [16]. HPLC chromatograms of the Friedel-Crafts products are included in the Supplementary Materials.

\section{Conclusions}

The chiral aziridine-phosphines proved to be an effective catalyst for the asymmetric Friedel-Crafts alkylation of indoles with $\beta$-nitrostyrenes in the presence of the copper(I) complex and triethylamine. The appropriate Friedel-Crafts products were formed in satisfactory chemical yields and with high enantiomeric excess values. The absolute configuration of the carbon atom of aziridine has a decisive influence on the stereochemical course of the title reaction. 
Supplementary Materials: The following are available online at http://www.mdpi.com/2073-4344/10/9/971/s1: copies of NMR spectra of compounds 1-8 and selected HPLC chromatograms for Friedel-Crafts products.

Author Contributions: Conceptualization and methodology, S.L. and M.R.; software, A.B., A.Z. and M.R.; investigation, A.B., A.Z.; writing-original draft preparation, M.R.; writing-review and editing, S.L. and M.R.; supervision, M.R. and S.L. All authors have read and agreed to the published version of the manuscript.

Funding: This research was funded by the National Science Centre (NCN) (Grant No. 2016/21/B/ST5/00421 for M.R.).

Conflicts of Interest: The authors declare no conflict of interest.

\section{References}

1. Da Gama Oliveira, V.; do Carmo Cardoso, M.F.; da Silva Magalhães Forezi, L. Organocatalysis: A brief overview on its evolution and application. Catalysts 2018, 8, 605. [CrossRef]

2. Pellissier, H. Asymmetric organocatalysis. Tetrahedron 2007, 63, 9267-9331. [CrossRef]

3. Kagan, H.B.; Gopalaiah, K. Early history of asymmetric synthesis: Who are the scientists who set up the basic principles and the first experiments. New J. Chem. 2011, 35, 1933-1937. [CrossRef]

4. Li, L.; Chen, Z.; Zhang, X.; Jia, Y. Divergent strategy in natural product total synthesis. Chem. Rev. 2018, 118, 3752-3832. [CrossRef]

5. Shimokawa, J. Divergent strategy in natural product total synthesis. Tetrahedron Lett. 2014, 55, 6156-6162. [CrossRef]

6. Krautwald, S.; Carreira, E.M. Stereodivergence in asymmetric catalysis. J. Am. Chem. Soc. 2017, 139, 5627-5639. [CrossRef]

7. Choi, J.; Fu, G.C. Catalytic asymmetric synthesis of secondary nitriles via stereoconvergent Negishi arylations and alkenylations of racemic $\alpha$-bromonitriles. J. Am. Chem. Soc. 2012, 134, 9102-9105. [CrossRef]

8. Kalek, M.; Fu, G.C. Phosphine-catalyzed doubly stereoconvergent $\gamma$-additions of racemic heterocycles to racemic allenoates: The catalytic enantioselective synthesis of protected $\alpha, \alpha$-disubstituted $\alpha$-amino acid derivatives. J. Am. Chem. Soc. 2015, 137, 9438-9442. [CrossRef] [PubMed]

9. Park, J.K.; Lackey, H.H.; Ondrusek, B.A.; McQuade, D.T. Stereoconvergent synthesis of chiral allylboronates from an E/Z mixture of allylic aryl ethers using a 6-NHC-Cu(I) catalyst. J. Am. Chem. Soc. 2011, 133, 2410-2413. [CrossRef] [PubMed]

10. Poulsen, T.B.; Jørgensen, K.A. Catalytic asymmetric Friedel-Crafts alkylation reactions-Copper showed the way. Chem. Rev. 2008, 108, 2903-2915. [CrossRef] [PubMed]

11. Singh, P.K.; Bisai, A.; Singh, V.K. Enantioselective Friedel-Crafts alkylation of indoles with nitroalkanes catalyzed by a bis(oxazoline)-Cu(II) complex. Tetrahedron Lett. 2007, 48, 1127-1129. [CrossRef]

12. Li, W. Chiral bis(oxazolinyl)thiophenes for enantioselective $\mathrm{Cu}$ (II)-catalyzed Friedel-Crafts alkylation of indole derivatives with nitroalkenes. Catal. Lett. 2014, 144, 943-948. [CrossRef]

13. Liu, T.-Y.; Cui, H.-L.; Chai, Q.; Long, J.; Li, B.-J.; Wu, Y.; Ding, L.-S.; Chen, Y.-C. Organocatalytic asymmetric Friedel-Crafts alkylation/cascade reactions of naphthols and nitroolefins. Chem. Commun. 2007, 2228-2230. [CrossRef] [PubMed]

14. Wang, Y.-Q.; Song, J.; Hong, R.; Li, H.; Deng, L. Asymmetric Friedel-Crafts reaction of indoles with imines by an organic catalyst. J. Am. Chem. Soc. 2006, 128, 8156-8157. [CrossRef] [PubMed]

15. Özdemir, H.S.; Şahin, E.; Çakici, M.; Kiliç, H. Asymmetric Friedel-Crafts alkylation of pyrrole with nitroalkenes catalyzed by a copper complex of a bisphenol A-derived Schiff base. Tetrahedron 2015, 71, 2882-2890. [CrossRef]

16. Kim, H.Y.; Kim, S.; Oh, K. Orthogonal enantioselectivity approaches using homogeneous and heterogeneous catalyst systems: Friedel-Crafts alkylation of indole. Angew. Chem. Int. Ed. 2010, 49, 4476-4478. [CrossRef]

17. Liu, J.; Gong, L.; Meggers, E. Asymmetric Friedel-Crafts alkylation of indoles with 2-nitro-3-arylacrylates catalyzed by a metal-templated hydrogen bonding catalyst. Tetrahedron Lett. 2015, 56, 4653-4656. [CrossRef]

18. Kaushik, N.K.; Kaushik, N.; Attri, P.; Kumar, N.; Kim, C.H.; Verma, A.K.; Choi, E.H. Biomedical importance of indoles. Molecules 2013, 18, 6620-6662. [CrossRef] 
19. Sakamoto, T.; Itoh, J.; Mori, K.; Akiyama, T. Chiral Brønsted acid catalyzed Friedel-Crafts alkylation reaction of indoles with $\alpha, \beta$-unsaturated ketones: Short access to optically active 2- and 3-substituted indole derivatives. Org. Biomol. Chem. 2010, 8, 5448-5454. [CrossRef]

20. Ali, S.; Wisal, A.; Tahir, M.N.; Ali, A.; Hameed, S.; Ahmed, M.N. One-pot synthesis, crystal structure and antimicrobial activity of 6-benzyl-11-(p-tolyl)-6H-indolo[2,3-b]quinoline. J. Mol. Struct. 2020, 1210, 128035. [CrossRef]

21. Sansinenea, E.; Martínez, E.F.; Ortiz, A. Organocatalytic synthesis of chiral spirooxindoles with quaternary stereogenic centers. Eur. J. Org. Chem. 2020. [CrossRef]

22. Hong, S.K.; Park, W.; Park, Y.S. Asymmetric synthesis of 4-aryl dihydroisoquinolin-3-ones and 2-aryl morpholin-3-ones using AgOTf-activated $\alpha$-bromo arylacetate. Tetrahedron 2020, 76, 130841. [CrossRef]

23. Wang, Z.; Zu, L. Organocatalytic enantioselective direct alkylation of phloroglucinol derivatives: Asymmetric total synthesis of (+)-aflatoxin B2. Chem. Commun. 2019, 5171-5174. [CrossRef]

24. Yang, H.; Tang, W. Efficient enantioselective syntheses of chiral natural products facilitated by ligand design. Chem. Rec. 2020, 20, 23-40. [CrossRef]

25. Khatri, H.R.; Carney, N.; Rutkoski, R.; Bhattarai, B.; Nagorny, P. Recent progress in steroid synthesis triggered by the emergence of new catalytic methods. Eur. J. Org. Chem. 2020, 7, 755-776. [CrossRef]

26. Zhao, Y.-L.; Lou, Q.-X.; Wang, L.-S.; Hu, W.-H.; Zhao, J.-L. Organocatalytic Friedel-Crafts alkylation/Lactonization reaction of naphthols with 3-trifluoroethylidene oxindoles: The asymmetric synthesis of dihydrocoumarins. Angew. Chem. Int. Ed. 2017, 56, 338-342. [CrossRef]

27. Roemer, M.; Wild, D.A.; Sobolev, A.N.; Skelton, B.W.; Nealon, G.L.; Piggott, M.J.; Koutsantonis, G.A. Carbon-rich trinuclear octamethylferrocenophanes. Inorg. Chem. 2019, 58, 3789-3799. [CrossRef]

28. Doğan, Ö.; Çağli, E. PFAM catalyzed enantioselective diethylzinc addition to imines. Turk. J. Chem. 2015, 39, 290-296. [CrossRef]

29. Dogan, Ö.; Tan, D. Enantioselective direct aldol reactions promoted by phosphine oxide aziridinyl phosphonate organocatalysts. Tetrahedron Asymmetry 2015, 26, 1348-1353. [CrossRef]

30. Eröksüz, S.; Dogan, Ö.; Garner, P.P. A new chiral phosphine oxide ligand for enantioselective 1,3-dipolar cycloaddition reactions of azomethine ylides. Tetrahedron Asymmetry 2010, 21, 2535-2541. [CrossRef]

31. Dogan, Ö.; Isci, M.; Aygun, M. New phosphine oxide aziridinyl phosphonates as chiral Lewis bases for the Abramov-type phosphonylation of aldehydes. Tetrahedron Asymmetry 2013, 24, 562-567. [CrossRef]

32. Dogan, Ö.; Bulut, A.; Tecimer, M.A. Chiral phosphine oxide aziridinyl phosphonate as a Lewis base catalyst for enantioselective allylsilane addition to aldehydes. Tetrahedron Asymmetry 2015, 26, 966-969. [CrossRef]

33. Wujkowska, Z.; Zawisza, A.; Leśniak, S.; Rachwalski, M. Phosphinoyl-aziridines as a new class of chiral catalysts for enantioselective Michael addition. Tetrahedron 2019, 75, 230-235. [CrossRef]

34. Buchcic, A.; Zawisza, A.; Leśniak, S.; Adamczyk, J.; Pieczonka, A.M.; Rachwalski, M. Enantioselective Mannich reaction promoted by chiral phosphinoyl-aziridines. Catalysts 2019, 9, 837. [CrossRef]

35. Leśniak, S.; Rachwalski, M.; Jarzyński, S.; Obijalska, E. Lactic acid derived aziridinyl alcohols as highly effective catalysts for asymmetric additions of an organozinc species to aldehydes. Tetrahedron Asymmetry 2013, 24, 1336-1340. [CrossRef]

36. Pieczonka, A.M.; Leśniak, S.; Rachwalski, M. Direct asymmetric aldol condensation catalyzed by aziridine semicarbazide zinc(II) complexes. Tetrahedron Lett. 2014, 55, 2373-2375. [CrossRef]

37. Pieczonka, A.M.; Marciniak, L.; Rachwalski, M.; Leśniak, S. Enantiodivergent aldol condensation in the presence of aziridine/acid/water systems. Symmetry 2020, 12, 930. [CrossRef]

38. Coumbe, T.; Lawrence, N.J.; Muhammad, F. Titanium (IV) catalysis in the reduction of phosphine oxides. Tetrahedron Lett. 1994, 35, 625-628. [CrossRef]

39. Zhang, T.-X.; Zhang, W.-X.; Luo, M.-M. Metal-free reduction of tertiary phosphine oxides with Hantzsch ester. Chin. Chem. Lett. 2014, 25, 176-178. [CrossRef]

40. Busacca, C.A.; Raju, R.; Grinberg, N.; Haddad, N.; James-Jones, P.; Lee, H.; Lorenz, J.C.; Saha, A.; Senanayake, C.H. Reduction of tertiary phosphine oxides with DIBAL-H. J. Org. Chem. 2008, 73, $1524-1531$. [CrossRef] [PubMed]

41. Provis-Evans, C.B.; Emanuelsson, E.A.C.; Webster, R.L. Rapid metal-free formation of free phosphines from phosphine oxides. Adv. Synth. Catal. 2018, 360, 3999-4004. [CrossRef]

42. Sowa, S.; Stankevič, M.; Szmigielska, A.; Małuszyńska, H.; Kozioł, A.E.; Pietrusiewicz, K.M. Reduction of functionalized tertiary phosphine oxides with BH3. J. Org. Chem. 2015, 80, 1672-1688. [CrossRef] [PubMed] 
43. Zhao, W.; Sun, J. Triflimide $\left(\mathrm{HNTf}_{2}\right)$ in organic synthesis. Chem. Rev. 2018, 118, 10349-10392. [CrossRef]

44. Shi, H.; Herron, A.N.; Shao, Y.; Shao, Q.; Yu, J.-Q. Enantioselective remote meta-C-H arylation and alkylation via a chiral transient mediator. Nature 2018, 558, 581-586. [CrossRef] [PubMed] 